\title{
INVESTIGACIÓN-ACCIÓN EN EL DESARROLLO DE PROYECTOS CURRICULARES INNOVADORES DE CIENCIAS
}

\author{
MEMBIELA, PEDRO \\ Didáctica Ciencias Experimentais de Facultade Ciencias da Educación \\ Universidade de Vigo. Edificio 1, as Lagoas, s/n. 32004 Ourense \\ membiela@uvigo.es
}

\begin{abstract}
Resumen. Un grupo de investigación-acción ha diseñado dos proyectos curriculares innovadores de ciencias para enseñanza secundaria, que tienen como meta fundamental la relevancia social y personal. El proceso de construcción curricular en la práctica ha estado orientado por la evaluación holística centrada en la mejora educativa, consiguiendo una valoración positiva por parte de los implicados. El análisis en profundidad de las condiciones educativas existentes y el uso de la investigación para el cambio en la escuela (Keeves, 1998) han supuesto, en nuestro caso, un importante desarrollo profesional de todos los integrantes del grupo de investigación-acción.

Palabras clave. Investigación-acción, proyectos curriculares innovadores, evaluación holística, desarrollo profesional.

Summary. An action-research group has designed two science innovative curriculum projects that have as their fundamental aim the social and personal relevance. The process of curriculum construction in the practice has been guided by the holistic evaluation centered in the educative improvement, getting a positive valuation on the part of those implied. The analysis in depth of the existent educational conditions and the use of investigation for the change in the school (Keeves, 1998), have implied in our case an important professional development of all the members of the action-research group.

Keyords. Action-research, innovative curriculum projects, holistic evaluation, professional development.
\end{abstract}

\section{INTRODUCCIÓN}

Los problemas de la formación científica de la población adulta y, por tanto, de la que se recibe en la escuela han sido denominados por algunos como crisis de alfabetización científica (Matthews, 1994), ante lo que Hodson (1993) ha sugerido llevar a cabo la educación científica más orientada socialmente y también más centrada en los estudiantes. En tal sentido se orienta la enseñanza de las ciencias para la responsabilidad social (Cross y Price, 1994; Jenkins, 1994; Ramsey, 1993) o el documento Ciencia para todos los estadounidenses (AAAS, 1989).

La educación en la ciencia para la acción, y para la relevancia social, tiene como objetivo ayudar a formar futuros ciudadanos para la acción y considera a los adolescentes como ciudadanos que pronto tendrán su lugar en la sociedad como adultos. Además, la relevan- cia personal se completa atendiendo a las necesidades actuales y no sólo futuras de los estudiantes. A un nivel más profundo está el compromiso para trabajar por una educación como agente de transformación de la sociedad y para mejorar las condiciones sociales del conjunto de la población.

En este marco teórico, un grupo de profesores de ciencias de un centro de enseñanza secundaria, apoyados y asesorados por personas vinculadas a la universidad, decidió formar un equipo de investigación-acción para diseñar, implementar y evaluar proyectos curriculares de ciencias orientados hacia la relevancia social y personal (Membiela, 1999). Estos proyectos curriculares son innovadores en España, fundamentalmente por las siguientes razones: $a$ ) porque son los propios profesores quienes diseñan el proyecto, incorporando de manera 
sistemática en todas las unidades de los proyectos temas de especial relevancia personal y social; $b$ ) porque los proyectos curriculares fueron puestos en práctica en contextos ordinarios, dentro de materias incluidas en el currículo oficial; $c$ ) porque los proyectos fueron evaluados, siguiendo un modelo holístico, que implica la valoración del diseño y del proceso de contrucción curricular en la práctica educativa; $d$ ) porque los profesores actúan también como investigadores; e) porque el proyecto nace por iniciativa de los profesores, sin que contaran inicialmente con ningún tipo de apoyo económico, lo que favoreció una gran autonomía de acción.

\section{FUNDAMENTACIÓN TEÓRICA}

\section{Investigación-acción como instrumento de cambio educativo}

Muchos intentos de reforma de la educación científica han utilizado los modelos denominados centro-periferia o de transmisión para el desarrollo curricular y han tenido poco éxito (Cuban, 1993). La clave parece estar en que dichas reformas educativas han olvidado la relación existente entre mejora curricular y desarrollo profesional (Van Den Akker, 1998). Por ello, actualmente se postula, para las reformas educativas, la investigaciónacción o el modelo de profesor como investigador (Lytle y Cochran-Smith, 1990).

La investigación-acción (IA) está interesada en los problemas prácticos que encuentran los profesores en las aulas y en encontrar soluciones a los mismos. Implica el desarrollo profesional de los profesores, y la idea de los profesores como investigadores ha sido ampliada para incorporar la teoría crítica en el análisis de las condiciones existentes en las escuelas y en el uso de la investigación con el fin de iniciar y promover cambio en las escuelas. Se producen diversos beneficios para los profesores cuando trabajan juntos haciendo investigación en sus escuelas: a) porque la investigación añade la teoría y el conocimiento base que los profesores necesitan para mejorar su práctica de enseñanza; $b$ ) hace a los profesores interesados y conscientes de los hallazgos de la investigación, porque se desarrolla en la escuela un clima de indagación y mejora sistemática; $c$ ) se crea un clima de colaboración entre todos los participantes para la mejora educativa; $d$ ) ayuda a identificar problemas y buscar soluciones de manera sistemática; y, por último, e) puede ser la base de los programas de formación permanente en las escuelas, que promueven mejoras prácticas y el posterior desarrollo de los profesores (Elliot, 1990; Keeves, 1998).

Esta experiencia se sitúa en la denominada investigación-acción colaboradora, que en el ámbito educativo suele agrupar personas vinculadas al mundo de las escuelas y de la universidad (Oja y Smulyan, 1989; Veal y Tippins, 1996). Tal enfoque coincide con otros casos (Hodson, 1999) en que un grupo de profesores, conociendo bien a los estudiantes, la localidad y el ambiente de centro, han trabajado juntos en cuestiones teóricas y prácticas relacionadas con el diseño y la implementación de un nuevo currículo científico en un ambiente crítico y de apoyo, y con la guía de facilitadores. Al unir los procesos de indagación, mejora de la práctica y desarrollo profesional, la investigación-acción ha servido para integrar enseñanza y desarrollo del profesorado, desarrollo del currículo y evaluación, investigación y reflexión, en una concepción unificada de la práctica reflexiva educativa.

\section{Investigación-acción en el desarrollo profesional de los profesores}

Actualmente en el ámbito del desarrollo profesional (Loucks-Horsley et al., 1998) se sugiere un cambio en el énfasis desde la transmisión de conocimiento al aprendizaje experiencial, desde la dependencia de los hallazgos previos de la investigación a examinar la propia práctica de enseñanza, desde el aprendizaje centrado en la persona al colaborativo y de la imitación de las mejores prácticas al aprendizaje centrado en los problemas. El desarrollo profesional debe ser visto como un proceso de cambio y renovación (Kyle, 1995), y se dice que IA puede contribuir al desarrollo profesional de los profesores (Berlin, 1996), integrándose en su práctica normal (Feldman, 1996) y dándoles el poder de tomar decisiones y aceptar responsabilidades en su propio desarrollo profesional (Loucks-Horsley et al., 1998). IA es considerada como una aproximación viable a la reforma y la mejora de la educación científica (Veal y Tippins, 1996), que tiene en cuenta la singularidad de cada situación educativa (Conelly y Clandinin, 1988) y, al darle a los profesores gran control sobre el currículo, crea el sentido de algo propio necesario en los cambios efectivos y a largo plazo (Hodson y Bencze, 1998). El desarrollo curricular se convierte en un vehículo para el desarrollo profesional y la reforma de las escuelas (Parke y Coble, 1997).

\section{EL PROCESO DE CONSTRUCCIÓN CURRI- CULAR EN LA PRÁCTICA EDUCATIVA}

Siguiendo las ideas de Stenhouse (1987), los proyectos se centran, más que en resultados, en la calidad del proceso educativo, a través de unos principios pedagógicos que actúan como criterios para seleccionar acciones docentes congruentes con las finalidades educativas de emancipación y comprensión. Nuestra concepción de currículo incluye una visión holística del mismo, ya que no sólo lo concebimos como una propuesta, un diseño, sino que también nos preocupamos por los procesos y productos no previstos, por las experiencias de aprendizaje y la organización de los contextos múltiples que estructuran y ofrecen las oportunidades de tales experiencias. Además, entendemos y utilizamos el currículo como una parcela de investigación e innovación educativa, así como una excelente oportunidad para la formación del profesorado en ejercicio.

En relación con lo que habitualmente se denomina implementación curricular, nos hemos situado en una 
perspectiva diferente de quienes se preocupan por el grado de fidelidad con que se pone en práctica el currículo previamente diseñado o de quienes se interesan por cómo se han adaptado las innovaciones. Nuestra orientación se centra en cómo se construye el currículo en la práctica (Snyder et al., 1992), entendido como un conjunto de experiencias educativas creadas por estudiantes y profesores, quienes adquieren un papel clave por su participación en la creación de conocimiento en la práctica o en el cambio curricular, entendido éste como un proceso de desarrollo individual y cambio en el pensamiento y en la práctica.

A continuación se señalan las principales líneas de diseño curricular, se describen ambos proyectos y se comenta su proceso de evaluación.

\section{Líneas de diseño curricular}

La incorporación de temas científicos de relevancia personal y social

Coincidiendo con algunas de las metas mencionadas por la National Science Teachers Association de Estados Unidos (Harms y Yager, 1981), ambos proyectos curriculares buscan potenciar el aprendizaje de las ciencias orientado a las necesidades personales, a preparar a los individuos para utilizar la ciencia en la mejora de sus propias vidas, y también aprender ciencia para colaborar en la resolución de los problemas sociales habituales, en conseguir ciudadanos informados, preparados para tratar con responsabilidad (Ramsey, 1993; Cross y Price, 1992) las cuestiones científicas con implicaciones sociales. En nuestro caso, y de acuerdo con Jenkins (1994), el aprendizaje de las ciencias amplía sus objetivos para ayudar a los estudiantes a desenvolverse en el ambiente social. Concedemos una especial importancia a las aportaciones del enfoque ciencia-tecnología-sociedad (Membiela, 1995, 1997; Yager, 1996), el cual pretende promover la alfabetización científica y técnica de los ciudadanos para que puedan participar en el proceso democrático de toma de decisiones y en la resolución de problemas relacionados con la ciencia y la tecnología. Este enfoque sugiere la selección de contenidos teniendo en cuenta los siguientes criterios: que sean aplicables a la vida actual de los estudiantes, que sean adecuados al desarrollo cognitivo y la madurez social de éstos, que sean temas importantes para el mundo actual-que lo sigan siendo en la futura vida adulta de los alumnos-y que sean contenidos por los que muestren interés y entusiasmo los estudiantes (Membiela, 1997).

\section{La incorporación sistemática de temáticas transversales}

Se integran de un modo sistemático las temáticas transversales en cada una de las unidades que los componen, de manera especial la educación ambiental, la educación para la salud y la educación para el consumo.

La educación ambiental pretende mejorar el conocimiento sobre los ambientes biofísicos y socioculturales del ser humano, concienciar sobre los problemas am- bientales y sus soluciones y motivar para actuar responsablemente (Jiménez y Laliena, 1992; Lucko et al., 1982). El proyecto curricular «La Naturaleza en la Ciudad» ha priorizado la educación ambiental en el territorio urbano de nuestra ciudad (Ourense), e incluye actividades en tal sentido a todo lo largo del currículo (por ejemplo, localizar vertederos incontrolados y evaluar sus posibles impactos ambientales) y una unidad didáctica denominada «Residuos sólidos urbanos» (Suárez et al., 1990).

La educación para la salud considera la escuela como un elemento esencial en la formación de valores, actitudes, hábitos, destrezas y conocimientos que capaciten a los estudiantes para responsabilizarse y saber tomar decisiones sobre su propia salud y la de sus semejantes (Nieda, 1992; Pollock, 1987). En la promoción de la salud de los adolescentes, la integración de la educación para la salud en el currículo de ciencias no se debe limitar a los aspectos curriculares sino que se debe incorporar el acceso a los servicios sanitarios y ser la escuela promotora de salud, aprovechando el interés que despiertan, en los estudiantes, los cambios corporales de la adolescencia (Crockett y Petersen, 1993). El proyecto curricular «La salud es cosa de todos» prioriza la educación para la salud e incluye actividades en tal sentido a todo lo largo del currículo (por ejemplo, sobre la utilización de los prospectos de los medicamentos) y una unidad didáctica denominada «La salud es cosa de todos», que se introduce como núcleo vertebrador de todo el proyecto.

En ambos proyectos también incorporamos tópicos de educación para el consumo con la finalidad de formar a los estudiantes como consumidores conscientes, críticos, responsables y solidarios (Jiménez, 1992; National Consumer Council, 1983), por ejemplo, analizando publicidad y elaborando contrapublicidad.

\section{La territorialización del currículo}

La educación debe ser un diálogo con la localidad (Orr, 1992, 1994; Sanger, 1997), importante porque combina pensamiento y acción, resuelve problemas de superespecialización y educa a la gente para percibir y utilizar los potenciales de su propia localidad. El medio próximo cumple importantes funciones educativas: en primer lugar, como factor de desarrollo y socialización de los estudiantes, que puede estimular su progreso planteándoles problemas e incitándolos a dar respuestas; y, en segundo lugar, como objeto de estudio, fuente de recursos materiales y humanos y excelente lugar de aprendizaje para la enseñanza de las ciencias. Por eso, hemos incluido, como temática, la ciudad de Ourense y sus problemas, sus ríos o sus fuentes termales.

\section{Otras líneas de diseño curricular}

El proceso de diseño se ha iniciado con la investigación acerca de las ideas previas sobre diversos temas relacionados con el medio ambiente y la salud (medio ambiente urbano, residuos sólidos urbanos, salud o alimentación) (Membiela et al., 1993a, 1993b, 1994; Dapía et al., 
1996a, 1996b) y, en nuestro caso conocer lo que piensan los estudiantes ha permitido mejorar el proceso de enseñanza-aprendizaje ayudando en el proceso de diseño de las actividades.

Se han incluido temas socialmente controvertidos (Wellington, 1986), definidos como tales porque implican juicios de valor y porque suponen debates en los medios de comunicación, entre expertos o entre ciudadanos. Estas controversias deben ser incluidas en la escuela, pues los estudiantes deben construir una opinión, fundada a través de evidencias documentales, de los problemas que se discuten socialmente, como el problema del hambre en el tercer mundo y en la ciudad de Ourense.

Aunque esté presente el medio próximo, también se integra la educación científica global, para que los estudiantes tomen conciencia de los problemas globales del mundo y de las repercusiones de sus acciones tanto individuales como colectivas (Pike y Selby, 1986), como por ejemplo, las repercusiones ambientales, políticas y económicas de la Guerra del Golfo.

La presencia de las denominadas etnociencias incorpora la cultura científica de las comunidades que nos son próximas, los saberes y creencias tradicionales ligados al mundo rural (agricultura, ganadería, cocina, medicina, artesanía, tecnología...). Frente a la exclusión total que la ciencia académica hace de las etnociencias, éstas son aprovechadas en la medida en que representan una parte del esfuerzo humano por comprender y actuar sobre el medio (Nader, 1996), como, por ejemplo, la utilidad de algunos remedios curativos tradicionales.

Junto a ello hay que señalar la presencia de una importante presencia de lo que podríamos denominar contenidos tradicionales de ciencias, como, por ejemplo, la célula, debido a la necesidad que tienen los estudiantes de superar años más tarde los exámenes de la selectividad y porque es necesario que profundicen en las perspectivas de la ciencia académica en la medida en que suponen la visión más racional del mundo.

\section{Descripción de los proyectos curriculares}

\section{La naturaleza en la ciudad}

Éste es el primer proyecto, que comenzó a ser diseñado en el curso 1988-89 y está destinado a alumnos de 12-14 años. Se caracteriza por incorporar, mediante la estrategia de infusión, temas de educación ambiental y, en menor medida, de otras áreas transversales.

Seis unidades componen el proyecto, y en cada una de ellas se trabajaban tópicos de educación ambiental, junto a contenidos tradicionales de biología. Entre otros, se incluyen aspectos como los que se indican a continuación:

Las plantas: tala de árboles para adorno navideño, sustitución del bosque autóctono, la industria de celulosas, las plantas trepadoras como pulmones de la ciudad. Los animales: tráfico ilegal de animales exóticos, pesca y caza furtivas, especies en extinción, maltrato de los animales. El clima: microclimas urbanos, el efecto invernadero, la lluvia ácida, el coche ecológico. Materiales geológicos: agotamiento de los recursos naturales, energías renovables, el petróleo como fuente de conflictos bélicos, la expoliación de la Amazonía por la extracción del uranio. El relieve: el agua como un bien escaso, detergentes con fosfato y eutrofización de las aguas, tratamiento de las aguas residuales, efectos sobre el suelo de los incendios forestales. Consumo y residuos sólidos urbanos: tratamiento de los residuos sólidos, vertederos incontrolados, residuos tóxicos, estrategias de reducción de las basuras.

\section{La salud es cosa de todos}

Éste es el segundo proyecto, que se inició en 1993, y que está dirigido a los estudiantes de ciencias de 14-16 años e incorpora, sobre todo, cuestiones relativas a la educación para la salud y algunos aspectos del resto de los temas transversales.

El proyecto consta de siete unidades; en ellas se incorporan, entre otros, los siguientes tópicos:

La salud es cosa de todos: concepto y determinantes de la salud, estilos de vida saludables, sistemas de atención sanitaria. El cuerpo humano, un mundo en miniatura: aguas embotelladas y bebidas deportivas, disoluciones para las lentes de contacto, aguas termales y mineromedicinales, enfermedades genéticas, publicidad de productos de belleza. Comer para vivir o vivir para comer: cultura culinaria tradicional, productos alimenticios industrializados, dietas equilibradas, consumo de alcohol, hambre y solidaridad, higiene bucodental y de los alimentos. Mantenimiento del cuerpo humano: medición de la temperatura corporal y la tensión arterial, primeros auxilios, tabaquismo, infartos cardíacos, análisis de sangre y orina, donación de órganos y trasplantes. Defendiéndonos: fabricación del yogur, enfermedades infecciosas, infecciones alimentarias, uso de los medicamentos. Sexualidad: sexualidad y cultura, mitos y prejuicios, discriminación sexual, sexualidad del adolescente, salud genital, autoexploración de los genitales, respuestas sexuales humanas, fecundación, embarazo, parto, anticoncepción, enfermedades de transmisión sexual, consumo publicitario y sexualidad, modelos estéticos, enfermedades específicas de la mujer. El cuerpo en movimiento: acné juvenil, alteraciones visuales, contaminación acústica, causas de lesiones medulares y vertebrales, drogodependencia, accidentes de tráfico.

Ambos proyectos han sido construidos conforme al modelo de diseño centrado en las actividades (Eisner, 1979), concretándose cada unidad del proyecto en una guía de actividades para el estudiante, que incluyen el material necesario para su realización, y otra guía con orientaciones didácticas para el profesor.

En los dos proyectos se utiliza, como estrategia mayoritaria de organización social del trabajo en el aula, el trabajo en pequeño grupo, seguido de debates colectivos 
y, en menor medida, de las actividades individuales y en gran grupo.

Los proyectos incluyen una amplia gama de actividades, siguiendo el principio de utilizar el tiempo de clase como un tiempo de aprendizaje y evitando la estrategia habitual de posponer el aprendizaje a los períodos de preparación de exámenes. Las actividades que se proponen son muy variadas, con diferentes grados de complejidad. Incluyen tareas como planteamiento de hipótesis, diseños o realización de actividades prácticas, comprobación experimental, observación directa, descripción e identificación, visitas, salidas e itinerarios, comentarios de textos, esquemas e ilustraciones, consulta bibliográfica, visionado y comentario de diapositivas y vídeos, elaboración o interpretación de tablas, gráficas y mapas, realización de esquemas, elaboración y comentario de dosieres, realización de informes, redacción de cartas, planificación y realización de campañas, expresión de preconcepciones y conocimientos previos, reflexiones sobre actitudes y comportamientos individuales, charlas de expertos, análisis de publicidad, juegos y talleres.

\section{Evaluación holística de los proyectos curriculares}

Simultáneamente con las tareas de diseñar, remodelar y poner en práctica las unidades, se llevaron a cabo una serie de investigaciones que tenían como objetivo dar respuesta a algunas dudas o problemas surgidos durante el proceso o simplemente mejorarlo. Esta dinámica generó un modelo holístico de evaluación de programas, en el que se ha intentado evaluar la calidad del currículo y de su construcción en la práctica, las condiciones de aprendizaje y, en menor grado, el aprendizaje conceptual, procedimental, actitudinal y comportamental de los estudiantes.

Las investigaciones realizadas utilizaron, en su mayoría, la triangulación de métodos y de perspectivas, con el objetivo de obtener una información lo más amplia y compleja posible de los aspectos evaluados. Así, en diversas investigaciones se utilizaron, para un mismo problema, diferentes instrumentos de recogida de información (el cuestionario, la entrevista y la información) y se recogió la opinión de los diferentes grupos implicados: los docentes, los estudiantes y los asesores o observadores externos.

La evaluación de los proyectos curriculares se focalizó en los siguientes aspectos:

- La utilización sistemática del trabajo en pequeños grupos (Nogueiras et al., 1994), que según la opinión de los estudiantes aporta interesantes ventajas: favorece el aprendizaje, desarrolla la capacidad de expresión, estimula las relaciones socioafectivas y hace la clase más activa y entretenida. Sin embargo, también detectaron, los estudiantes, algunos problemas, como, por ejemplo, el distinto grado de participación de los componentes del grupo. Los profesores, aunque coincidían en gran parte con las valoraciones de los estudiantes, percibían algunos problemas específicos, como, por ejemplo, que el ritmo de trabajo era lento o que el grado de participación y discusión disminuía a medida que transcurría el curso académico, y que les resultaba difícil conocer las aportaciones al grupo de cada estudiante concreto. Por otra parte, los observadores pusieron de manifiesto la elevada participación incluso de los estudiantes académicamente malos o mediocres, y que los estudiantes académicamente buenos actuaban como monitores de sus compañeros. El contraste de los diferentes puntos de vista nos ha permitido hacer una serie de reflexiones que más tarde sirvieron para introducir cambios en nuestra práctica educativa. En consecuencia, se buscaron y pusieron en práctica estrategias para corregir estos problemas e intentar mejorar el trabajo en equipo, tales como no formar equipos de trabajo fijos durante las dos primeras semanas de clase, para dejar que los estudiantes se conozcan un poco y facilitar así la formación de grupos naturales, realizar al comienzo del curso algún juego para favorecer el acercamiento personal o dar a aquellos estudiantes que lo deseen la posibilidad de realizar actividades individuales

- La evaluación del ambiente social de las aulas (Suárez et al., 1998) en las que se implementó el proyecto curricular fue positiva para los estudiantes, los profesores y los observadores externos, los cuales mostraron su satisfacción, sobre todo, en cuatro aspectos: la cooperación y cohesión de los estudiantes, tanto dentro de los pequeños grupos como en el grupo-clase; el aprovechamiento eficaz del tiempo de clase, que se utilizó como de aprendizaje real; el apoyo y la ayuda prestados por los profesores; y el carácter innovador de las actividades realizadas. Sin embargo, existían algunos aspectos que, aun cuando tenían una valoración aceptable, podían ser mejorados y deberían ser objeto de reflexión y de cambio. Nos referimos de manera singular a la implicación de los estudiantes. Los profesores y los observadores manifestaron que el interés de los estudiantes no era uniforme, pues dependía del tipo de actividades que realizaban (aumentaba en las manipulativas), de la hora del día en que se desarrollaba la clase (disminuía a última hora de la mañana, o por la tarde) y de la proximidad de los exámenes. A todo ello se debe añadir el cansancio de los estudiantes, producido por una jornada escolar de 67 horas diarias de clase. La utilización sistemática del trabajo en pequeño grupo parece fomentar un grado de afiliación relativamente alto, facilitando la socialización de los estudiantes, pero al mismo tiempo favorece un cierto desorden, que, si se mantiene dentro de unos límites, no debe ser entendido como sinónimo de desaprovechar el tiempo. En diseños curriculares relativamente cerrados, con actividades muy estructuradas, se produce una sensación de alta organización, pero al mismo tiempo parece afectar negativamente a la implicación de los estudiantes. Por ello, se introdujeron a lo largo del diseño curricular actividades abiertas, para dejar campo a la iniciativa de los estudiantes.

- En la evaluación de conjunto del proyecto curricular (Membiela et al., 1994), los estudiantes lo percibían como interesante, entretenido, agradable, participativo, variado, organizado, y al mismo tiempo un poco difícil. Valoraron muy positivamente los aprendizajes conse- 
guidos, sobre todo los referidos al conocimiento de su ciudad y a las cuestiones medioambientales, manifestando que, en su opinión, ampliaron sus conocimientos y su respeto e interés por la conservación del medio local, tomaron conciencia de sus problemas y modificaron su comportamiento. Los estudiantes también valoraron muy positivamente el dominio que los profesores tenían de la materia, reconociendo, así, el gran esfuerzo que habían realizado. La cuestión que recibió la valoración más baja (aunque aceptable) por parte de todos los participantes (profesores, alumnos y asesora externa) fue el sistema de evaluación, aunque por motivos diferentes. Los profesores manifestaron que el elevado número de estudiantes por clase (entre 35 y 40) y por profesor (entre 120 y 160) les hizo difícil poder obtener una amplia información de cada alumno, a través de los instrumentos de evaluación previstos: cuadernos de clase, observación del trabajo en grupo, actividades voluntarias y exámenes. La asesora externa fundamentó su opinión sobre el carácter disciplinario de la evaluación, en que a fin de cuentas la calificación clasifica, separa y jerarquiza a los individuos, por lo que no era de extrañar que fuera el aspecto más problemático en el proyecto curricular. Puesto que, según todas las opiniones, la evaluación constituía la principal fuente de insatisfacción, aun siendo un problema de difícil solución, se propusieron y, en cierta media, llevaron a la práctica procesos de negociación entre profesorado y estudiantes.

\section{A MODO DE CONCLUSIÓN}

En el marco teórico de la investigación-acción, un equipo de profesoras y asesores externos se ha encargado de la construcción de dos proyectos curriculares innovadores donde se han integrado múltiples líneas de diseño que tienen como meta fundamental la relevancia social y personal en el aprendizaje de ciencias.

Esta experiencia de investigación y desarrollo curricular se ha adaptado a una situación educativa concreta, por lo que las propuestas educativas se han ajustado mejor a los intereses y expectativas de las profesoras y los estudiantes, en un proceso cíclico y continuo de construcción del currículo en la práctica educativa, orientado por la evaluación holística centrada en la mejora educativa. En ese marco teórico ha sido positivo el análisis del proceso, a través de una serie de investigaciones sobre aspectos clave, tales como el trabajo en pequeño grupo de los estudiantes, el clima psicosocial del aula o el grado de satisfacción de los implicados -de manera singular estudiantes y profesores.

Por último, quisieramos destacar las repercusiones que todo el proceso de investigación tuvo sobre el desarrollo profesional de los profesores participantes, quienes ampliaron sus conocimientos, mejoraron sus habilidades profesionales -al ser agentes activos en el diseño, implementación y evaluación de un proyecto curricular-, adquirieron nuevos roles al actuar no sólo como profesores sino también como investigadores y lograron prestigio profesional al integrarse en la comunidad académica de innovación e investigación educativa.

\section{AGRADECIMIENTOS}

Esta investigación fue realizada gracias a la subvención concedida por la Xunta de Galicia, proyecto PGIDT00PXI39201PR.

\section{REFERENCIAS BIBLIOGRÁFICAS}

AAAS (1989). Project 2061. Science for all Americans. Washington, DC: AAAS.

BERLIN, D. F. (1996). Action research in the science classroom: Curriculum improvement and teacher professional development, en Rhoton, J. y Bowers, P. (eds.). Issues in Science Education, pp. 73-80. Arlington: NSELA/NSTA.

CONELLY, F. M. y CLANDININ, D. J. (1988). Teachers as curriculum planners: Narratives of experience. Nueva York: Teachers College Press.

CROCKETT, L.J. y PETERSEN, A.C. (1993). Adolescent development: Health risks and opportunities for health promotion, en Millstein, S.G., Petersen, A.C. y Nightingale, A.O.(eds). Promoting the health of adolescents: New directions for the twenty-first century. Oxford: Oxford University Press, pp. 13-37.

CROSS, R.T. y PRICE, R.F. (1992). Teaching science for social responsibility. Sydney: St. Louis Press.

CROSS, R. T. y PRICE, R .F. (1994). Scientific issues and social awareness: The case of biological diversity. School Science Review, 75(273), pp. 29-40.

CUBAN, L. (1993). How teachers taught: Constancy and change in American classrooms 1890-1990. Nueva York: Teachers College Press.

DAPÍA, M. D., CID, M. C. y MEMBIELA, P. (1996a) Utilización de las preconcepciones de los estudiantes acerca de la salud 
en el diseño, implementación y evaluación de una unidad didáctica. Investigación en la Escuela, 28, pp. 95-101.

DAPÍA, M.D., CID, M.C. y MEMBIELA, P. (1996b). Un proyecto curricular de ciencias orientado hacia la educación para la salud. Alambique, 9, pp. 57-62.

EISNER, E.W. (1979). He educational imagination: on the design and evolution of school programs. Nueva York: Macmillan.

ELLIOTT, J. (1990) El cambio educativo desde la investigaciónacción. Madrid: Morata.

FELDMAN, A. (1996). Enhancing the practice of physics teachers. Mechanisms for the generation and sharing of knowledge and understanding in collaborative action research. Journal of Research of Science Teaching, 33, pp. 513-540.

HARMS, J. y YAGER, R. (1981). What research says to the science teacher, 3. Washington: NSTA.

HODSON, D. (1993). In search of a meaningful relationship: an exploration of some issues relating to integration in science and science education. International Journal of Science Education, 14(5), pp. 541-562.

HODSON, D. (1999). Going beyond cultural pluralism: Science education for sociopolitical action. Science Education, 83, pp. 775-796.

HODSON, D. y BENCZE, L. (1998). Becoming critical about practical work: Changing views and changing practice through action research. International Journal of Science Education, 20, pp. 683-694.

JENKINS, E.W. (1994). Public understanding of science and science education for action. Journal of Curriculum Studies, (26) 6, pp. 601-611.

JIMÉNEZ, M.J. (1992) Temas transversales. Educación para el consumo. MEC: Madrid.

JIMÉNEZ, M.J. y LALIENA, L. (1992) Temas transversales. Educación ambiental. MEC: Madrid.

KEEVES, J.P. (1998). Methods and processes in research in science education, en Fraser, B.J. y Tobin, K.G. (eds.). International handbook of science education, pp. 11271155. Dordrecht: Kluwer.

KYLE, W.C. (1995). Professional development: The growth and learning of teachers as professional over time. Journal of Research in Science Teaching, 32, pp. 679-681.

LAYTON, D., JENKINS, E., MACGILL, S. y DAVEY, A. (1993). Inarticulate science? Nafferton, UK: Studies in Education.

LOUCKS-HORSLEY, S., HEWSON, P., LOVE, N. y STILES, K.E. (1998). Designing professional development for teachers of science and mathematics. Corwin: Thousand Oaks.

LUCKO, B., DESINGER, J. y ROTH, R. (1982). Evaluation of environmental education programs at the elementary and secondary school levels. Journal of Environmental Education, 13(4), pp. 7-14.

LYTLE, S. y COCHRAN-SMITH, M. (1990). Learning from teacher research: A working typology. Teachers College Record, 92, pp. 83-103.

MATTHEWS, M.R. (1994) Science Teaching: The Role of History a Philosophy of Science. Londres: Routledge.
MEMBIELA, P. (1995). Ciencia-tecnología-sociedad en la enseñanza-aprendizaje de las ciencias experimentales. Alambique, 3, pp. 7-12.

MEMBIELA, P. (1997). Una revisión del movimiento educativo ciencia-tecnología-sociedad. Enseñanza de las Ciencias, 15(1), pp. 51-58.

MEMBIELA, P. (1999). Towards the reform of science teaching in Spain: the social and personal relevance of junior secondary school science projects for a broader understanding of science. International Journal of Science Education, 21, pp.721730 .

MEMBIELA, P., NOGUEIRAS, E. y SUÁREZ, M. (1993a). Students' preconceptions about environmental problems in cities, with particular reference to solid urban waste. Journal of Environmental Education, 24(2), pp. 30-34.

MEMBIELA, P., NOGUEIRAS, E. y SUÁREZ, M. (1993b). Concepciones previas de los estudiantes sobre algunos temas ambientales relacionados con el agua. Investigación en la Escuela, 20, pp. 81-90.

MEMBIELA, P., NOGUEIRAS, E. y SUÁREZ, M. (1994). Alternative conceptions of students about the natural urban environment in a small Spanish city. The Environmentalist, 14(2), pp. 131-138.

MEMBIELA, P., SUÁREZ, M. y NOGUEIRAS, E. (1994). Evaluación del proyecto naturaleza en la ciudad. Enseñanza de las Ciencias, 12(1), pp. 22-29.

NADER, L. (1996). Naked science. Nueva York: Routledge.

NATIONAL CONSUMER COUNCIL (1983). A better class of consumer. An investigation into consumer education in secondary schools. Londres: National Consumer Council.

NIEDA, J. (1992). Temas transversales. Educación para la salud. Educación sexual. Madrid: MEC.

NOGUEIRAS, E., MEMBIELA, P. y SUÁREZ, M. (1994) Triangulando perspectivas: el trabajo en grupo a debate. Revista de Educación, 302, pp. 259-271.

OJA, S.N., y SMULYAN, L. (1989). Collaborative action research: A developmental approach. Lewes: Falmer Press.

ORR, D. (1992). Ecological literacy. Education and the transition to a postmodern world. Nueva York: Suny Press.

ORR, D. (1994). Earth in mind. Washington: Island Press.

PARKE, H.M. y COBLE, C.R. (1997). Teachers designing curriculum as professional development: A model for transformational science teaching. Journal of Research in Science Teaching, 34(8), pp. 773-790.

PIKE, G. y SELBY, D. (1986). Global education, en Wellington, J.J. (ed.). Controversial issues in the curriculum, pp. 39-58. Oxford: Blackwell,

POLLOCK, M. (1987). Planning and implementing health education in schools. Palo Alto: Mayfield.

RAMSEY, J. (1993). The science education reform movement: Implications for social responsibility. Science Education, 77(2), pp. 235-258.

SANGER, M. (1997). Sense of Place and Education. The Journal of Environmental Education, 29, pp. 4-8.

SNYDER, J., BOLIN, F. y ZUMWALT, K. (1992). En Jackson, P.W. (ed.) Handbook of research on curriculum, pp. 402435. Nueva York: Macmillan. 
STENHOUSE, L. (1987). La investigación como base de la enseñanza. Madrid: Morata.

SUÁREZ, M., NOGUEIRAS, E., MEMBIELA, P., LATORRE, P., CID, M.C. y CAMBA, M.X. (1990). Consumo y residuos sólidos urbanos. Cuadernos de Pedagogía, 186, pp. 58-62.

SUÁREZ, M., PÍAS, R., MEMBIELA, P. y DAPÍA, D. (1998). Classroom environment in the implementation of an innovative curriculum project in science education. Journal of Research in Science Teaching, 35(6), pp. 655-671.

VAN DEN AKKER, J. (1998). The science curriculum: Between ideals and outcomes, en Fraser, B.J. y Tobin, K.G. (eds.).
International handbook of science education, pp. 421-449. Dordrecht: Kluwer.

VEAL, W.R., y TIPPINS, D. J. (1996). Action research: Creating a context for science teaching and learning, en Rhoton, J. y Bowers, P. (eds.). Issues in Science Education, pp. 81-87. Arlington: NSELA/NSTA.

WELLINGTON, J.J. (1986) Introduction, en Wellington, J.J. (ed.). Controversial issues in the curriculum, pp. 1-5. Oxford: Blackwell.

YAGER, R.E. (ed.) (1996). Science/Technology/Society as Reform in Science Education. Nueva York: State University of New York Press.

[Artículo recibido en mayo de 2001 y aceptado en enero de 2002.] 